\title{
Prevalence and phenotypic correlations of EIF1AX mutations in thyroid nodules
}

\author{
Arivarasan Karunamurthy, Federica Panebianco, Susan J Hsiao, Jennie Vorhauer, \\ Marina N Nikiforova, Simion Chiosea and Yuri E Nikiforov
}

Department of Pathology, University of Pittsburgh Medical Center, Pittsburgh, Pennsylvania, USA

Correspondence should be addressed

to Y E Nikiforov

Email

nikiforovye@upmc.edu

\begin{abstract}
The EIF1AX gene mutations have been recently found in papillary thyroid carcinoma (PTC) and anaplastic thyroid carcinoma (ATC). The prevalence of these mutations in other types of thyroid cancers and benign nodules is unknown. In this study, we analyzed the occurrence of EIF1AX mutations in exons 2, 5, and 6 of the gene in a series of 266 thyroid tumors and hyperplastic nodules by either Sanger or next-generation sequencing (ThyroSeq v.2). In addition, 647 thyroid fine-needle aspiration (FNA) samples with indeterminate cytology were analyzed. Using surgically removed samples, EIF1AX mutations were detected in 3/86 (2.3\%) PTC, 1/4 (25\%) ATC, 0/53 follicular carcinomas, 0/12 medullary carcinomas, 2/27 (7.4\%) follicular adenomas, and 1/80 (1.3\%) hyperplastic nodules. Among five mutation-positive FNA samples with surgical follow-up, one nodule was PTC and others were benign follicular adenomas or hyperplastic nodules.

Overall, among 33 mutations identified, A113_splice mutation at the intron 5/exon 6 splice site of EIF1AX was the most common. All four carcinomas harbored A113_splice mutation and three of them had one or more coexisting mutations, typically RAS. All PTC carrying EIF1AX mutations were encapsulated follicular variants. In summary, this study shows that EIF1AX mutations occur not only in thyroid carcinomas, but also in benign nodules. The most common mutation hotspot is the A113_splice, followed by a cluster of mutations in exon 2. When found in thyroid FNA samples, EIF1AX mutations confer $\sim 20 \%$ risk of cancer; the risk is likely to be higher in nodules carrying a A113_splice mutation and when EIF1AX coexists with RAS mutations.
\end{abstract}

\section{Key Words \\ - EIF1AX \\ thyroid \\ - FNA \\ - papillary carcinoma \\ - follicular adenoma}

\section{Introduction}

Thyroid cancer is the most common endocrine cancer and its incidence is increasing in the USA and other countries due to improved detection of indolent thyroid cancers by sensitive imaging techniques and possibly due to other factors (Davies \& Welch 2006, Brito et al. 2013, Jung et al. 2014). Cytological diagnosis of thyroid nodules using cells collected by fine-needle aspiration (FNA) is the most reliable way of cancer diagnosis, but a significant proportion of FNA samples is placed in indeterminate cytological category, preventing optimal patient management (Haugen et al. 2016). Advances in sequencing technology coupled with better understanding of the molecular mechanisms of thyroid tumorigenesis have led to increasing use of genomic testing for clinical management of patients with thyroid nodules (Adeniran et al. 2006, Cantara et al. 2010, Nikiforov et al. 2011). Recently, The Cancer Genome Atlas (TCGA) study on a large cohort of papillary thyroid carcinomas (PTC) reported mutations of the EIF1AX gene in these tumors (Cancer Genome Atlas Research 2014c).

Published by Bioscientifica Ltd 
The EIF1AX gene located on chromosome $\mathrm{X}$ and codes for an eukaryotic translation initiation factor 1A (eIF1A). The protein promotes $43 \mathrm{~S}$ complex formation by stabilizing the binding of the ternary complex eIF2-GTP-methionylinitiator tRNA to the 40S subunit and, together with other translation initiation factor is involved in a sophisticated scanning system responsible for accurately locating the proper start codon on the mRNA in eukaryotes (Pestova et al. 2001, Olsen et al. 2003, Fekete et al. 2005, Hinnebusch 2014). eIF1A is the eukaryotic ortholog of bacterial translation initiation factor IF1, with sequences between 32 and 95 amino acids constituting the RNA-binding domain that are homologous to IF1. Additional domains in eIF1, absent in IF1, are a helical domain adjacent to the RNAbinding fold and long and highly charged unstructured $\mathrm{N}$ - and C-terminal tails (NTT, CTT). The CTT is primarily negatively charged but contains a few hydrophobic residues at the very end that were proposed to be involved in protein-protein interactions (Fekete et al. 2007, Saini et al. 2010). Mutations of residues on the RNA-binding surface of eIF1A are reported to cause defects in proper $43 \mathrm{~S}$ and $48 \mathrm{~S}$ preinitiation complex formation (Battiste et al. 2000). Prior to TCGA study of PTC, mutations in EIF1AX gene were reported in uveal melanomas (Martin et al. 2013, Ewens et al. 2014). A recent study also reported EIF1AX mutations in anaplastic thyroid carcinomas (ATC) (Kunstman et al. 2015). However, the prevalence of these mutations in other common types of thyroid cancer, such as follicular carcinoma, and in benign thyroid nodules remains unknown. Furthermore, histopathologic characteristics of thyroid tumors harboring EIF1AX mutations are not well characterized. The aim of this study was to determine the prevalence of EIF1AX mutations in all major types of thyroid tumors and benign nodules, spectrum of EIF1AX mutation, and histopathologic characteristics of thyroid lesions harboring EIF1AX mutations.

\section{Materials and methods}

\section{Study cases}

This study was approved by the University of Pittsburgh Institutional Review Board. Two groups of samples were studied. The first group included 266 surgical specimens (formalin-fixed paraffin-embedded and snap-frozen tissue samples) that were used to study the prevalence of EIF1AX mutations in various types of thyroid nodules. The second group included 647 consecutive fine-needle aspiration (FNA) samples with indeterminate cytology genotyped during a 2-month time interval.
Table 1 Prevalence of EIF1AX mutations in thyroid nodules.

\begin{tabular}{|c|c|c|}
\hline Histopathologic diagnosis & Total cases & $\begin{array}{l}\text { EIF1AX mutation } \\
\text { positive }(\%)\end{array}$ \\
\hline $\begin{array}{l}\text { Papillary thyroid } \\
\text { carcinoma }\end{array}$ & 86 & $2(2.3)$ \\
\hline $\begin{array}{l}\text { Follicular thyroid } \\
\text { carcinoma }\end{array}$ & 53 & 0 \\
\hline $\begin{array}{l}\text { Poorly differentiated } \\
\text { thyroid carcinoma }\end{array}$ & 4 & 0 \\
\hline $\begin{array}{l}\text { Anaplastic thyroid } \\
\text { carcinoma }\end{array}$ & 4 & $1(25)$ \\
\hline $\begin{array}{l}\text { Medullary thyroid } \\
\text { carcinoma }\end{array}$ & 12 & 0 \\
\hline Follicular adenoma & 27 & $2(7.4)$ \\
\hline Hyperplastic nodule & 80 & $1(1.3)$ \\
\hline
\end{tabular}

\section{Histologic review}

Glass slides of surgical resection of all cases positive for EIF1AX mutation were retrieved from the archives of the Department of Pathology, University of Pittsburgh Medical Center and reviewed to confirm histopathologic diagnosis and tumor variant, and to assess for other salient features.

\section{Molecular analysis}

Testing for EIF1AX mutations in exons 2, 5, and 6 was performed using Sanger sequencing or next-generation sequencing panel (ThyroSeq v2) as previously described (Nikiforov et al. 2014). In addition to EIF1AX, the panel included the analysis of point mutations in the hotspots of 13 other thyroid cancer-related genes (AKT1, BRAF, NRAS, HRAS, KRAS, PTEN, TP53, GNAS, CTNNB1, RET, PIK3CA, TERT, and TSHR) and gene fusions in the RET, BRAF, NTRK1, NTRK3, ALK, PPARG, and THADA genes. For some samples, Sanger sequencing was performed on ABI13130 (Applied Biosystems) for exons 2, 5, and 6 of EIF1AX gene. For targeted next-generation sequencing analysis, the custom primers were designed using a Life

Table 2 Location and type of EIF1AX mutations found in thyroid nodules.

\begin{tabular}{|c|c|c|c|}
\hline $\begin{array}{l}\text { Mutated } \\
\text { codon }\end{array}$ & Frequency (\%) & $\begin{array}{l}\text { Mutation } \\
\text { variants }\end{array}$ & $\begin{array}{l}\text { Coexisting } \\
\text { mutations }\end{array}$ \\
\hline Codon 113 & $15(46)$ & A113_splice & $\begin{array}{c}\text { NRAS, HRAS, } \\
\text { TP53 }\end{array}$ \\
\hline Codon 9 & $8(24)$ & G9R, G9D, G9V & \\
\hline Codon 13 & $4(12)$ & $\begin{array}{l}\text { R13L, R13P, } \\
\text { R13D }\end{array}$ & NRAS, GNAS \\
\hline Codon 8 & $2(6)$ & G8R, G8V & - \\
\hline Codon 15 & $2(6)$ & G15D & \\
\hline Codon 10 & $1(3)$ & K10N & \\
\hline Codon 6 & $1(3)$ & G6_splice & - \\
\hline
\end{tabular}

Published by Bioscientifica Ltd 


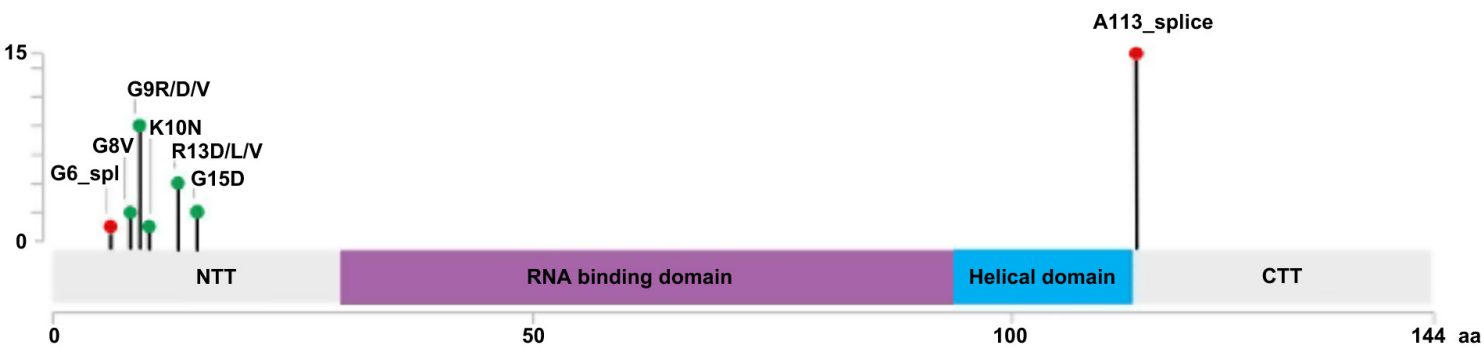

\section{Figure 1}

Spectrum of EIF1AX mutations found in thyroid nodules. Distribution of EIF1AX mutations within various domains of eukaryotic IF1A protein (144 amino acids) identified in various types of thyroid lesions. X-axis denotes the number of mutations. Green circles denotes missense mutations and red circles denotes splice-site mutations. A full colour version of this figure is available at http://dx.doi.org/10.1530/ERC-16-0043.

Technologies design tool to generate a pool of primers for amplification of genomic regions of interest. Library concentration and amplicon size were determined using an Agilent BioAnalyzer High Sensitivity DNA Kit (Agilent Technologies). Next, multiplexed barcoded libraries were enriched by clonal amplification using emulsion PCR on Ion Sphere particles (Ion PGM Template OT2 200 Kit, Thermo Fisher Scientific) and loaded on an Ion 318 Chip. Massively parallel sequencing was carried out on a Personal Genome Machine sequencer (Ion Torrent) using the Ion PGM Sequencing 200 Kit version 2 according to the manufacturer's instructions.

\section{Results}

First, the prevalence of EIF1AX mutation was assessed in a series of surgically resected thyroid nodules representing all common types of thyroid cancer. Among 86 surgical PTC samples, two specimens were positive for EIF1AX mutations, resulting in an overall frequency of $2.3 \%$. Both mutations were a splice-site mutation located in the inton 5/exon 6 junction of EIF1AX gene (A113_splice). Analysis of eight dedifferentiated carcinomas (including four poorly differentiated carcinomas and four anaplastic carcinomas) identified an A113_splice mutation in one ATC. Analysis of 53 follicular thyroid carcinomas (FTC), which included 22 oncocytic (Hürthle cell) variants of follicular carcinomas, did not reveal EIF1AX mutations. Similarly, no EIF1AX mutation was identified in 12 medullary carcinomas. Screening of benign thyroid nodules detected EIF1AX mutations in two (7.4\%) follicular adenomas (FA) and one (1.3\%) hyperplastic thyroid nodules (Table 1).

Next, the analysis was performed on 647 consecutive thyroid FNA cytology samples with indeterminate cytology, of which 27 (4.2\%) were found to be positive for EIF1AX mutations. Of the EIF1AX-positive cytology specimens, 63\% had a diagnosis of atypia of undetermined significance/ follicular lesion of undetermined significance, 30\% had a diagnosis of follicular neoplasm/suspicious for follicular neoplasm, and $4 \%$ had a diagnosis of suspicious for malignancy. Follow-up thyroidectomy specimens were available in 5 of the 27 samples. Histopathologically, the EIF1AX-positive nodules yielded the diagnosis of PTC in one case, follicular adenoma in three cases, and hyperplastic thyroid nodule in one case. Out of 27 thyroid nodules positive for EIF1AX mutations in FNA samples, 21 nodules had no coexisting

Table 3 Clinicopathological characteristics of thyroid nodules harboring EIF1AX mutations and coexisting mutations.

\begin{tabular}{|c|c|c|}
\hline Case number & Diagnosis & Age \\
\hline 1 & EFVPTC & 34 \\
\hline 2 & EFVPTC & 72 \\
\hline 3 & EFVPTC & 69 \\
\hline 4 & ATC & 71 \\
\hline 5 & FA & 46 \\
\hline 6 & FA & 45 \\
\hline 7 & FA & 69 \\
\hline 8 & FA & 77 \\
\hline 9 & $\mathrm{FA}$ & 68 \\
\hline 10 & $\mathrm{HN}$ & 63 \\
\hline 11 & $\mathrm{HN}$ & 53 \\
\hline
\end{tabular}

\begin{tabular}{c} 
Gender \\
\hline $\mathrm{M}$ \\
$\mathrm{M}$ \\
$\mathrm{M}$ \\
$\mathrm{F}$ \\
$\mathrm{F}$ \\
$\mathrm{F}$ \\
$\mathrm{F}$ \\
$\mathrm{F}$ \\
$\mathrm{F}$ \\
$\mathrm{M}$ \\
$\mathrm{F}$
\end{tabular}

\begin{tabular}{c} 
Nodule size $(\mathrm{cm})$ \\
\hline 5.1 \\
6.0 \\
1.5 \\
6 \\
2.5 \\
2.9 \\
1.8 \\
1.3 \\
3.5 \\
4.3 \\
2.7
\end{tabular}

\begin{tabular}{l} 
EIF1AX mutation type \\
\hline A113_splice \\
A113_splice \\
A113_splice \\
A113_splice \\
K10N \\
G9R \\
G9V \\
A113_splice \\
A113_splice \\
G9R \\
G9R
\end{tabular}

\begin{tabular}{l} 
Coexisting mutation \\
\hline NRAS \\
NRAS \\
- \\
NRAS, TP53, TERT \\
- \\
- \\
- \\
- \\
- \\
- \\
-
\end{tabular}

EFVPTC, encapsulated follicular variant of papillary thyroid carcinoma; HN, hyperplastic nodule. 

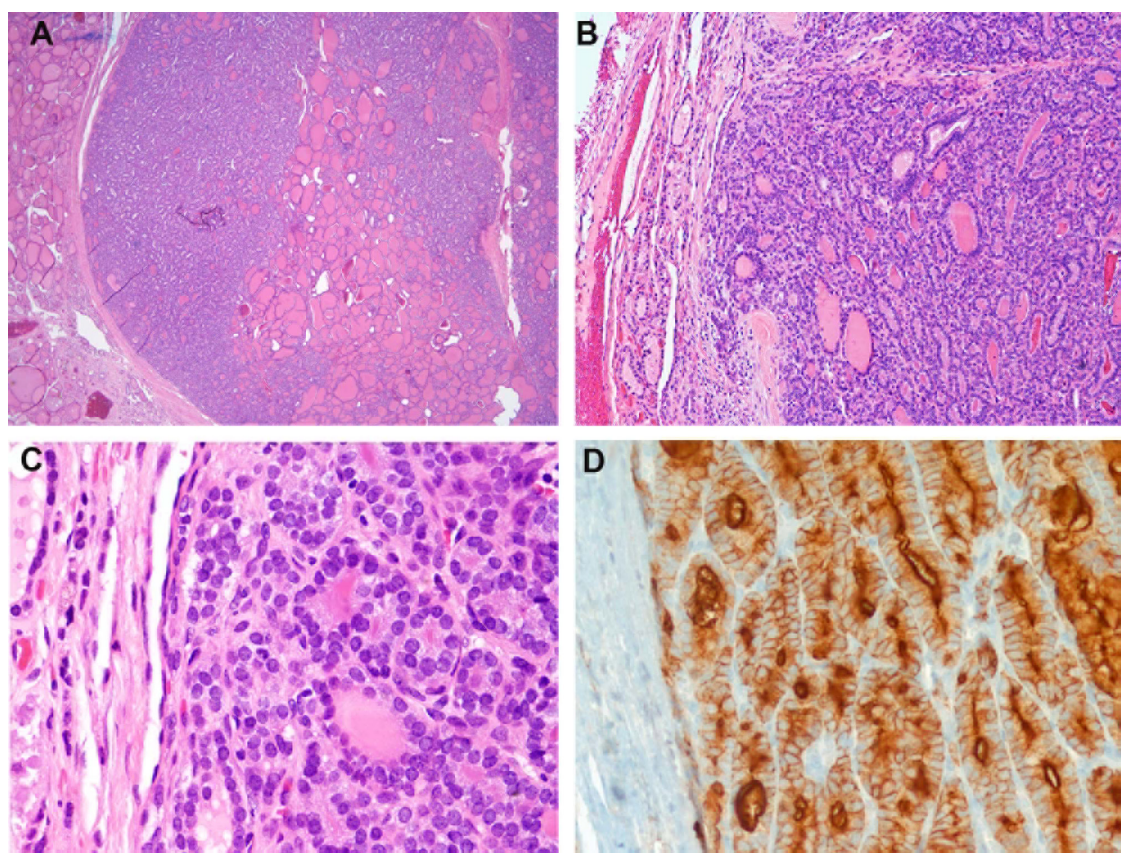

\section{Figure 2}

Histopathologic features of papillary thyroid carcinomas positive for EIF1AX mutations. Encapsulated follicular variant of papillary thyroid carcinoma with well-defined capsule and mixed microfollicular and normofollicular growth pattern (A) and capsular invasion (B). The neoplastic follicular cells show enlarged, overlapping, irregularly shaped nuclei (C) and strong and diffuse membranous immunoreactivity for HBME-1 (D). A, hematoxylin and eosin (H\&E) stain $\times 20 ; B, H \& E, \times 100 ; C, H \& E$, $\times 40$; D, HBME-1 immunostain, $\times 100$.

mutations, whereas 6 nodules had additional mutations. Of those, four nodules showed presence of a coexisting NRAS codon 61 mutation, one sample had a HRAS codon 61 mutation, and one had a GNAS mutation. Of these six cases, one nodule harboring EIF1AX and NRAS mutation was surgically excised to reveal the encapsulated follicular variant of PTC.

Overall, in the two sample groups, 33 EIF1AX mutations were identified (Table 2). All mutations were single nucleotide substitutions that were clustered in two areas of the gene, either in codons 6-15 located in the unstructured NTT (54\%) or codon 113 corresponding to the unstructured CTT (46\%) of the EIF1AX (Fig. 1). Codon 113 was split by intron 5 and harbored the most common EIF1AX mutation identified in 15 (46\%) of EIF1AX-positive cases. These were splice-site mutations affecting one of the two consensus intronic nucleotides (c.338-1A and c.338-2G) located in the splice site of intron 5/exon 6. The second most common mutation, found in eight (24\%) cases, affected codon 9, leading to the substitution of glycine by arginine, aspartic acid, or valine. EIF1AX codon 13 missense mutations were identified in four (12\%) cases and resulted in the amino acid substitution of arginine to leucine, aspartic acid, or proline. Less common mutations affected codons 8 , 10 , and 15 , all being missense mutations, while a single case showed a splice-site mutation at codon 6 , which was split by intron 1 .

A total of 11 cases that harbored EIF1AX mutation were available for histopathology review (Table 3). The size of these thyroid nodules ranged from 1.3 to $6.0 \mathrm{~cm}$ (mean, $2.9 \mathrm{~cm}$ ) and histopathologic diagnoses included PTC in three cases, ATC in one case, follicular adenoma in five cases, and hyperplastic thyroid nodule in five cases (Table 3).

All three mutation-positive PTCs carried the same EIF1AX A113_splice mutation. Two of these tumors also harbored a coexisting mutation of NRAS. The tumor nodules ranged in size from 1.5 to $6.0 \mathrm{~cm}$. Microscopically, they had a complete thin or thick capsule and follicular growth pattern characteristic of the encapsulated follicular variant of PTC (Fig. 2). The neoplastic follicles in each nodule varied in size with abundant colloid and were lined by cells showing moderate to prominent nuclear features of PTC. A tumor with isolated EIF1AX mutation showed focal capsular invasion (Fig. 2), whereas other tumors showed no tumor capsule or vascular invasion. None of the PTCs carrying the EIF1AX mutation had extrathyroidal extension or lymph node metastasis.

One ATC case was positive for the EIF1AX A113_splice mutation found in combination with NRAS Q61R, TERT C228T, and TP53 E180* stop codon mutation. Microscopic examination of the excised tumor revealed a combination of epithelioid, spindle cell, and multinucleated giant cell growth patterns and large areas of necrosis. No welldifferentiated component was observed.

Five FA were found to harbor EIF1AX mutations, including A113_splice site $(\times 2), \mathrm{G} 9 \mathrm{R} / \mathrm{V}(\times 2)$, and $\mathrm{K} 10 \mathrm{~N}$. The nodules ranged in size from 1.3 to $3.5 \mathrm{~cm}$, had a thin to thick capsule, and typically a mixture of small- to normal-sized follicles (Fig. 3A and B). Two adenomas were

Published by Bioscientifica Ltd 

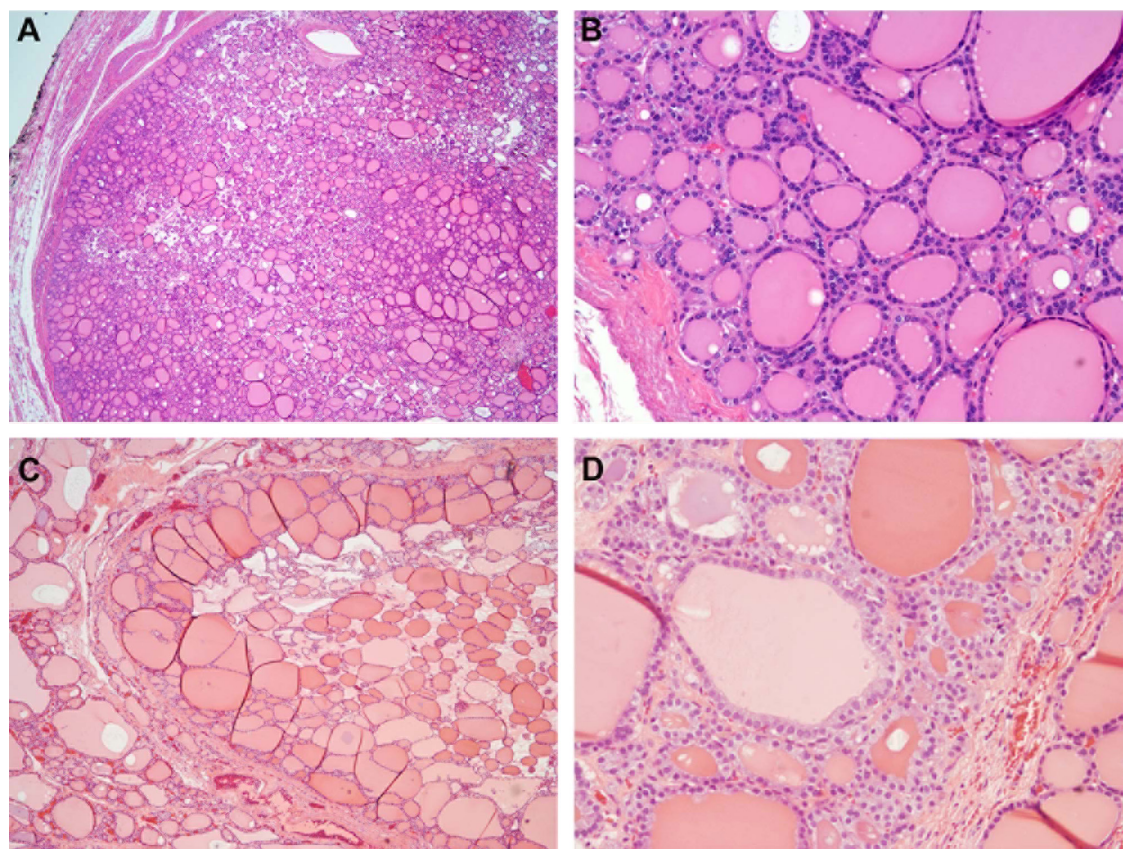

\section{Figure 3}

Histopathologic features of EIF1AX-positive benign thyroid nodules. (A, B) A follicular adenomas surrounded by a thin fibrous capsule (A) and showing small- to normal-sized follicles lined by cells with round, uniform nuclei and dark chromatin. (A, H\&E, ×20; $B, H \& E, \times 400)$. (C, D) Hyperplastic thyroid nodule with a thin capsule and a mixture of normal- and large-sized follicles with abundant colloid. (C, H\&E, x20; D, $H \& E, \times 400)$. A full colour version of this figure is available at http://dx.doi.org/10.1530/ERC-160043. of conventional type and one showed oncocytic (Hürthle cell) morphology. Two additional EIF1AX-positive nodules were diagnosed as hyperplastic nodules. Both of them harbored a G9R EIF1AX mutation. The nodules had a thin capsule and were composed of predominantly large follicles with abundant colloid (Fig. 3C and D).

\section{Discussion}

The results of this study confirm the presence of EIF1AX mutations in PTC and ATC and demonstrate for the first time that these mutations also occur in benign thyroid nodules. Furthermore, we show that EIF1AX mutations may coexist with other driver mutations such as RAS and TP53, which is found more frequently in malignant nodules.

The frequency of EIF1AX mutations in PTC found in this study is comparable to that reported by TCGA (Cancer Genome Atlas Research 2014c), indicating that EIF1AX mutations are reproducibly found in $1-2 \%$ of these tumors. Most of the EIF1AX-mutated PTCs (5/6 cases; $83 \%$ ) in TCGA study were registered as follicular variants (Gao et al. 2013). The sixth PTC case also showed follicular components, but with predominant classic PTC features explained by the presence of BRAF V600E mutation in addition to KRAS Q61K and EIF1AX mutations. Similarly, in our study all three PTCs were follicular variants, and all were encapsulated. This indicates that the follicular variant of PTC, typically encapsulated, is a typical phenotype of EIF1AX-mutated PTC.
Since the follicular variant of PTC is known to share genetic alterations with other follicular-patterned thyroid tumors, that is, FTC and FA (Nikiforov \& Nikiforova 2011), it was important to understand if EIF1AX mutations also occur in FTC and FA. Our analysis revealed no EIF1AX mutations in any of 53 FTC, but identified them in 7\% of FA. Even though a complete absence of EIF1AX mutations in FTC cannot be ruled out, these results suggest that the prevalence of EIF1AX mutations in FTC is likely to be low. The finding of these mutations in FA raises a theoretical possibility that EIF1AX-mutated FA have a propensity to progress to the follicular variant of PTC rather than to FTC.

EIF1AX mutations appear to frequently coexist with other mutations, particularly RAS. In this study, co-occurrence of NRAS mutation was observed in three of four EIF1AX-mutated cancers, but not in any benign thyroid nodules. Similarly, combination of EIF1AX and $R A S$ mutations was observed in previously reported PTC and ATC (Cancer Genome Atlas Research 2014c, Kunstman et al. 2015), and in other types of epithelial cancer and melanomas (Cerami et al. 2012, Gao et al. 2013, Cancer Genome Atlas Research 2014a,b). This raises a possibility that EIF1AX mutations alone are not sufficient for full transformation, but requires other mutations, particularly $R A S$, for progression to overt malignancy.

We identified a total of 33 samples harboring EIF1AX mutations that were clustered at exon 2 and intron 5 splice site. The two consensus nucleotides within intron 5 (c.338-1A, c.338-2G) immediately upstream to the exon 6 splice site is the most common and recurrent hotspot

Published by Bioscientifica Ltd 
for mutations. Of interest, four out of six thyroid nodules carrying this mutation were cancers in our study. Similar hotspot splice site mutation was also reported in two of six PTCs in TCGA study and two of four ATCs by Knutsman et al. (Cancer Genome Atlas Research 2014c, Kunstman et al. 2015, Chai et al. 2016). The EIF1AX A113_splice mutation affects the splicing of EIF1AX transcripts at the boundary of the helical domain within the C-strand and the highly charged unstructured CTT downstream involved in protein-protein interactions (Saini et al. 2010). Residues in the NTT and CTT of eIF1A act in opposite manner on scanning and start codon recognition and mutations in both NTT and CTT residues have been reported to impair preinitiation complex (PIC) assembly (Fekete et al. 2005, Saini et al. 2010). Nevertheless, an exact functional significance of codon 113 splice-site mutation that preserves RNA-binding domain, but altering the C-strand and CTT domains remains unclear.

Other common hotspot for EIF1AX mutations is exon 2 , which codes for the unstructured NTT domain. Codon 9 mutations are most commonly affected at this site. Mutations involving codons 13, 10, 15, and intron 1/exon 2 splice site were also identified in this study, and those have not been previously reported in thyroid tumors. Mutations in exon 2 coding for the NTT segments were found to promote an open scanning conducive conformation of PIC leading to leaky scanning, but these mutations were located between residues 17-21 of the gene (Fekete et al. 2005, Saini et al. 2010). In this study, all nodules carrying a mutation affecting exon 2 were benign. However, exon 2 mutations were found to occur in four PTC in the TCGA study (Cancer Genome Atlas Research 2014c). Overall, the available data suggest that the EIF1AX p.A113_splice mutation confers a higher risk of cancer in a given nodule than the exon 2 mutations.

It is important to stress that not all patients with thyroid nodules underwent surgery, and therefore the histologic outcomes for those nodules were not available. This represents a limitation of the study.

In summary, we report here the occurrence of EIF1AX mutations not only in thyroid cancer, but also in benign thyroid nodules, and demonstrate that phenotypically these mutations are associated with the encapsulated follicular variant of PTC and benign follicular-pattern nodules. Furthermore, the presence of A113_splice mutation, particularly in combination with RAS mutation, is more frequently associated with thyroid cancer than isolated EIF1AX mutations or mutations located at other hotspots of the gene.
Declaration of interest

YE N is a consultant for Quest Diagnostics. All other authors have nothing to disclose.

\section{Funding}

This work was supported in part by the NIH grant CA88041, funds from the University of Pittsburgh Cancer Institute and University of Pittsburgh Medical Center, and by the Richard A and Leslie A Snow Fund for Thyroid Cancer Research.

\section{References}

Adeniran AJ, Zhu Z, Gandhi M, Steward DL, Fidler JP, Giordano TJ, Biddinger PW \& Nikiforov YE 2006 Correlation between genetic alterations and microscopic features, clinical manifestations, and prognostic characteristics of thyroid papillary carcinomas. American Journal of Surgical Pathology 30 216-222.

Battiste JL, Pestova TV, Hellen CU \& Wagner G 2000 The eIF1A solution structure reveals a large RNA-binding surface important for scanning function. Molecular Cell 5 109-119. (doi:10.1016/S10972765(00)80407-4)

Brito JP, Yarur AJ, Prokop LJ, McIver B, Murad MH \& Montori VM 2013 Prevalence of thyroid cancer in multinodular goiter versus single nodule: a systematic review and meta-analysis. Thyroid 23 449-455. (doi:10.1089/thy.2012.0156)

Cancer Genome Atlas Research Network 2014a Comprehensive molecular characterization of gastric adenocarcinoma. Nature 513 202-209. (doi:10.1038/nature13480)

Cancer Genome Atlas Research Network 2014b Comprehensive molecular profiling of lung adenocarcinoma. Nature $511543-550$ (doi:10.1038/nature13385)

Cancer Genome Atlas Research Network 2014c Integrated genomic characterization of papillary thyroid carcinoma. Cell 159 676-690. (doi:10.1016/j.cell.2014.09.050)

Cantara S, Capezzone M, Marchisotta S, Capuano S, Busonero G, Toti P Di Santo A, Caruso G, Carli AF, Brilli L, et al. 2010 Impact of protooncogene mutation detection in cytological specimens from thyroid nodules improves the diagnostic accuracy of cytology. Journal of Clinical Endocrinology and Metabolism 95 1365-1369. (doi:10.1210/ jc.2009-2103)

Cerami E, Gao J, Dogrusoz U, Gross BE, Sumer SO, Aksoy BA, Jacobsen A, Byrne CJ, Heuer ML, Larsson E, et al. 2012 The cBio cancer genomics portal: an open platform for exploring multidimensional cancer genomics data. Cancer Discovery 2 401-404. (doi:10.1158/2159-8290.CD-12-0095)

Chai L, Li J \& Lv Z 2016 An integrated analysis of cancer genes in thyroid cancer. Oncology Reports 35 962-970. (doi:10.3892/ or.2015.4466)

Davies L \& Welch HG 2006 Increasing incidence of thyroid cancer in the United States, 1973-2002. JAMA 295 2164-2167. (doi:10.1001/jama.295.18.2164)

Ewens KG, Kanetsky PA, Richards-Yutz J, Purrazzella J, Shields CL, Ganguly T \& Ganguly A 2014 Chromosome 3 status combined with BAP1 and EIF1AX mutation profiles are associated with metastasis in uveal melanoma. Investigative Ophthalmology \& Visual Science $\mathbf{5 5}$ 5160-5167. (doi:10.1167/iovs.14-14550)

Fekete CA, Applefield DJ, Blakely SA, Shirokikh N, Pestova T, Lorsch JR \& Hinnebusch AG 2005 The eIF1A C-terminal domain promotes initiation complex assembly, scanning and AUG selection in vivo. EMBO Journal 24 3588-3601. (doi:10.1038/sj.emboj.7600821)

Fekete CA, Mitchell SF, Cherkasova VA, Applefield D, Algire MA Maag D, Saini AK, Lorsch JR \& Hinnebusch AG 2007 N- and 
C-terminal residues of eIF1A have opposing effects on the fidelity of start codon selection. EMBO Journal 26 1602-1614. (doi:10.1038/ sj.emboj.7601613)

Gao J, Aksoy BA, Dogrusoz U, Dresdner G, Gross B, Sumer SO, Sun Y, Jacobsen A, Sinha R, Larsson E, et al. 2013 Integrative analysis of complex cancer genomics and clinical profiles using the cBioPortal. Science Signaling 6 pl1. (doi:10.1126/ scisignal.2004088)

Haugen BR, Alexander EK, Bible KC, Doherty GM, Mandel SJ, Nikiforov YE, Pacini F, Randolph GW, Sawka AM, Schlumberger M, et al. 20162015 American thyroid association management guidelines for adult patients with thyroid nodules and differentiated thyroid cancer: The American thyroid association guidelines task force on thyroid nodules and differentiated thyroid cancer. Thyroid 26 1-133. (doi:10.1089/thy.2015.0020)

Hinnebusch AG 2014 The scanning mechanism of eukaryotic translation initiation. Annual Review of Biochemistry 83 779-812. (doi:10.1146/annurev-biochem-060713-035802)

Jung CK, Little MP, Lubin JH, Brenner AV, Wells SA Jr., Sigurdson AJ \& Nikiforov YE 2014 The increase in thyroid cancer incidence during the last four decades is accompanied by a high frequency of BRAF mutations and a sharp increase in RAS mutations. Journal of Clinical Endocrinology and Metabolism 99 E276-E285. (doi:10.1210/ jc.2013-2503)

Kunstman JW, Juhlin CC, Goh G, Brown TC, Stenman A, Healy JM, Rubinstein JC, Choi M, Kiss N, Nelson-Williams C, et al. 2015 Characterization of the mutational landscape of anaplastic thyroid cancer via whole-exome sequencing. Human Molecular Genetics $\mathbf{2 4}$ 2318-2329. (doi:10.1093/hmg/ddu749)

Martin M, Masshofer L, Temming P, Rahmann S, Metz C, Bornfeld N, van de Nes J, Klein-Hitpass L, Hinnebusch AG, Horsthemke B, et al.
2013 Exome sequencing identifies recurrent somatic mutations in EIF1AX and SF3B1 in uveal melanoma with disomy 3. Natural Genetics 45 933-936. (doi:10.1038/ng.2674)

Nikiforov YE, Carty SE, Chiosea SI, Coyne C, Duvvuri U, Ferris RL, Gooding WE, Hodak SP, LeBeau SO, Ohori NP, et al. 2014 Highly accurate diagnosis of cancer in thyroid nodules with follicular neoplasm/suspicious for a follicular neoplasm cytology by ThyroSeq v2 next-generation sequencing assay. Cancer 120 3627-3634. (doi:10.1002/cncr.29038)

Nikiforov YE \& Nikiforova MN 2011 Molecular genetics and diagnosis of thyroid cancer. Nature Reviews Endocrinology 7 569-580. (doi:10.1038/nrendo.2011.142)

Nikiforov YE, Ohori NP, Hodak SP, Carty SE, LeBeau SO, Ferris RL, Yip L, Seethala RR, Tublin ME, Stang MT, et al. 2011 Impact of mutational testing on the diagnosis and management of patients with cytologically indeterminate thyroid nodules: a prospective analysis of 1056 FNA samples. Journal of Clinical Endocrinology and Metabolism 96 3390-3397. (doi:10.1210/jc.2011-1469)

Olsen DS, Savner EM, Mathew A, Zhang F, Krishnamoorthy T, Phan L \& Hinnebusch AG 2003 Domains of eIF1A that mediate binding to eIF2, eIF3 and eIF5B and promote ternary complex recruitment in vivo. EMBO Journal 22 193-204. (doi:10.1093/ emboj/cdg030)

Pestova TV, Kolupaeva VG, Lomakin IB, Pilipenko EV, Shatsky IN, Agol VI \& Hellen CU 2001 Molecular mechanisms of translation initiation in eukaryotes. PNAS 98 7029-7036. (doi:10.1073/ pnas.111145798)

Saini AK, Nanda JS, Lorsch JR \& Hinnebusch AG 2010 Regulatory elements in eIF1A control the fidelity of start codon selection by modulating tRNA(i)(Met) binding to the ribosome. Genes and Development 24 97-110. (doi:10.1101/gad.1871910)

Received in final form 22 February 2016

Accepted 24 February 2016

Accepted Preprint published online 24 February 2016
(C) 2016 Society for Endocrinology Printed in Great Britain
Published by Bioscientifica Ltd 